\title{
A STRUCTURAL CHARACTERIZATION OF EXTENDED CORRECTNESS-COMPLETENESS IN CLASSICAL LOGIC
}

\author{
José ALFREdo AMOR \\ Facultad de Ciencias \\ Universidad Nacional Autónoma de México \\ jaam@hp.fciencias.unam.mx
}

SUMMARY: In this paper I deal with first order logic and axiomatic systems. I present the metalogical results that show the property of satisfying Modus Ponens as a necessary and sufficient condition for the extended completeness of the system, and to the Deduction Metatheorem as a necessary and sufficient condition for the extended correctness of the system. Both supposing that the system satisfies the corresponding restricted properties. These results show that the choice of that rule of inference and of that metatheorem, for any particular axiomatic system, are not a matter of personal liking or of practical convenience, but they play a fundamental role for the extended correctnesscompleteness properties of the axiomatic system. As a matter of fact, they can be considered as structural properties that characterize the fulfilling of the Extended Correctness and Completeness theorem for the axiomatic system.

KEY WORDS: logical consequence, axiomatic system, compactness, semantics

RESUMEN: En este artículo trabajo con la lógica de primer orden y presento dos resultados metalógicos respecto a sistemas axiomáticos que satisfacen la propiedad de correctud-completud restringida. El primer resultado relaciona la propiedad de que el sistema satisfaga Modus Ponens como condición necesaria y suficiente para la completud extendida del sistema. El segundo relaciona la propiedad de que el sistema satisfaga el Metateorema de la Deducción como condición necesaria y suficiente para la correctud extendida del sistema. Estos resultados muestran que la elección de esa regla de inferencia y de ese metateorema, para un sistema axiomático particular, no son sólo cuestión de gusto personal o de conveniencia práctica, sino que desempeñan un papel fundamental para la correctud-completud extendida del sistema axiomático. De hecho, pueden considerarse como propiedades estructurales que caracterizan que el sistema axiomático cumpla la correctud y la completud extendidas.

PALABRAS CLAVE: consecuencia lógica, sistema axiomático, compacidad, semántica 


\section{Introduction}

In what follows I will only work with formal first order languages with equality. I will deal with the concept of first order classical deductive logic, particularly with the concept of an axiomatic system of Hilbert type that is founded in the concepts of axiom, rule of inference and definition of formal derivation. As comes next I give the definition of what I will understand by an axiomatic system of Hilbert type.

Definition. An axiomatic system $\mathbf{S}$ is given by the following:

a) A finite or infinite decidable ${ }^{1}$ set $\Delta$, of formulas. The formulas of $\Delta$ are called the axioms of $\mathbf{S}$.

b) A finite set RI of decidable ${ }^{2}$ rules of inference of $\mathbf{S}$.

c) A definition of formal derivation of a formula $\alpha$ from a set of formulas $\Sigma$, in such a way that the derivation be a finite list of $n$ formulas $\alpha_{1}, \ldots, \alpha_{n}$, with $n \geq 1$, such that $\alpha_{n}=\alpha$ and for all $i(i=1, \ldots, n)$, either $\alpha_{i}$ is an axiom of $\mathbf{S}$ or $\alpha_{i}$ is a formula of $\Sigma\left(\alpha_{i}\right.$ is a hypothesis of $\left.\Sigma\right)$, or $\alpha_{i}$ is obtained from earlier formulas in the list by virtue of some rule of inference of the set RI of the rules of inference of $\mathbf{S}$. There may or may not be restrictions to the application of such a rule and in case there are some, these must be effectively decidable. If such a derivation exists, that is denoted by $\Sigma \vdash_{\mathbf{S}} \alpha$ and is read " $\alpha$ is derivable from $\Sigma$ in the system $\mathbf{S}$ ".

In Section 2 I will show the compactness theorem and some basic semantic properties that consider only the classical notions of truth and of logical consequence, ${ }^{3}$ as well as some basic syntactical properties that only consider the definition of axiomatic

${ }^{1}$ For each formula, an effective procedure must be given in order to decide if the formula is or is not an axiom.

${ }^{2}$ For each rule, an effective procedure must be given to decide if a given formula is or is not a consequence, of other formulas by virtue of that rule.

${ }^{3}$ I am referring to Tarski's definition of truth, which will not be developed here, but can be read in Mendelson 1987, chapter two. The intuitive idea is that a sentence $\varphi$ is true with respect to an interpretation (or model) $\mathfrak{A}$, if it is the case that the interpretation of $\varphi$ is a fact in $\mathfrak{A}$. [Tarski 1935.] 
system and that are therefore fulfilled by any axiomatic system. In Section 3 I present the objective of this paper: the metalogical results mentioned above and their proofs justified only with the compactness theorem and the basic properties of the earlier section. Finally, in Section 4 I give some conclusions.

\section{Basic Properties}

Semantics relates formal languages with an interpretation for them. In my case of a first order language with equality, this interpretation is a structure consisting of a non-empty set called the universe, together with relations, operations and distinguished objects from the set. Interpretations are to be understood in the standard way of model theory. A formula $\varphi$ is true in an interpretation $\mathfrak{A}$ if it is satisfied with respect to all sequences (or variable-assignments) in that interpretation. ${ }^{5}$

Before I continue working with the concept of logical consequence, I consider to make it precise here. Let $\Sigma \cup\{\varphi\}$ be a set of formulas, not necessarily sentences, in a first order language with equality.

Definition. I say that $\varphi$ is a logical consequence of $\Sigma$ (written $\Sigma \vDash \varphi$ ) if and only if in every interpretation $\mathfrak{A}$ every sequence $s$ (of elements of the universe of $\mathfrak{A}$ ) that satisfies $\alpha$ for every $\alpha \in \Sigma$, also satisfies $\varphi$. $^{6}$

For example, I have that $\forall x P(x) \vDash P(c)$ but $P(x) \not \forall \forall x P(x)$ and that $\varphi \vDash \forall x \varphi$ implies $\vDash(\varphi \rightarrow \forall x \varphi)$, for any formula $\varphi$, not necessarily a sentence.

I will use some basic semantic properties ${ }^{7}$ whose proofs are elementary and depend only on the definitions of truth and logical consequence. These are the following:

a) If $\Gamma \subseteq \Sigma$ and $\Gamma \vDash \varphi$, then $\Sigma \vDash \varphi$ (Monotony).

b) If $\Sigma \vDash \varphi$ and for all $\alpha$ in $\Sigma, \Gamma \vDash \alpha$, then $\Gamma \vDash \varphi$ (Cut).

${ }^{4}$ Cf. Enderton 2001, p. 80 or Mendelson 1987, p. 46.

${ }^{5}$ Cf. Enderton 2001, p. 87 or Mendelson 1987, p. 48.

${ }^{6}$ Cf. Mendelson 1987, p. 52 and Enderton 2001, p. 88.

${ }^{7}$ These are also called structural properties of classical deductive logic. 
c) $\Sigma, \alpha \vDash \beta$ if and only if $\Sigma \vDash(\alpha \rightarrow \beta)$.

d) $\Sigma \vDash \varphi$ if and only if $\Sigma \cup\{\neg \varphi\}$ is not satisfiable.

e) $\Sigma, \alpha,(\alpha \rightarrow \beta) \vDash \beta$.

Property c) for $\Sigma$ empty, establishes the equivalence between the notion of logical consequence and that of logical validity for the corresponding implicative formula. ${ }^{8}$ Property d) is a rewriting of the definition of logical consequence. If the language does not have the symbol " $\rightarrow$ " of material implication, I will understand that $(\alpha \rightarrow \beta)$ to be an abbreviation for the formula $(\neg \alpha \vee \beta)$ or for some formula that represents the corresponding truth function.

Some of these semantic properties will be used, from now on, appealing to any of them only as "semantic properties".

If the concept of " $\varphi$ is a logical consequence of $\Gamma$ " was characterized as " $\varphi$ is true in every interpretation that makes every formula in $\Gamma$ true", this concept is strictly weaker than the given above in the sense that my concept implies this one but not conversely. On the other hand, with this concept of course the corresponding clause c) of section 2 would fail. In the case that all formulas are sentences, the two concepts are equivalent. These facts are easily verified.

I will use the compactness theorem for first order languages with equality, which I consider a fundamental non-elementary semantic result and I present it in the form I will use it.

Compactness Theorem (Gödel-Malcev). If $\Sigma \cup\{\varphi\}$ is a set of formulas of a first order language with equality and $\Sigma \vDash \varphi$, then there is a finite subset $\Gamma \subseteq \Sigma$, such that $\Gamma \vDash \varphi$.

Notice that the compactness theorem is a purely semantic assertion; it does not involve deductions at all. It involves only semantic notions and I have a purely semantic proof for it, on

${ }^{8}$ Cf. Mendelson 1987, p. 52 and Enderton 2001, pp. 88, 99. 
the basis of semantic properties. I give an outline of the proof of an equivalent form: ${ }^{9}$

I start with the set of formulas $\Sigma$ such that every finite subset is satisfiable (call this property "finitely satisfiable"). Two previous results are proven, the first one consists in getting a set $\Gamma$ such that $\Sigma \subseteq \Gamma$ and $\Gamma$ is a maximal finitely satisfiable set of formulas, to prove this, Zorn Lemma is used. The second result consists in getting a set $\Omega$ such that $\Sigma \subseteq \Omega, \Omega$ is closed under existential "witness" 10 and is a finitely satisfiable set of formulas. Then the two processes are iterated by recursion and the union of all those sets is a set $\Sigma^{*}$ such that $\Sigma \subseteq \Sigma^{*}$ and $\Sigma^{*}$ is a maximal closed under witness and finitely satisfiable set of formulas. Then a model is constructed for $\Sigma^{*}$. That model is obviously a model for $\Sigma$ and I have $\Sigma$ satisfiable.

I show now some basic syntactic properties that refer to axiomatic systems, whose proof is elementary because it depends only on the very definition of axiomatic system and then they don't depend on which axiomatic system $\mathbf{S}$ one is dealing with. These are the following:

a) If $\Gamma \subseteq \Sigma$ and $\Gamma \vdash_{\mathrm{s}} \varphi$, then $\Sigma \vdash_{\mathrm{S}} \varphi$ (Monotony).

b) If $\Sigma \vdash_{\mathbf{S}} \varphi$ and for all $\alpha$ in $\Sigma, \Gamma \vdash_{\mathbf{S}} \alpha$, then $\Gamma \vdash_{\mathbf{S}} \varphi$ (Cut).

Note that these two properties are the syntactic counterparts of the corresponding first two semantic properties presented above.

Another elementary property is reflexivity; that is, $\alpha \vdash_{\mathbf{S}} \alpha$. Observe that transitivity, that is:

$$
\text { if } \alpha \vdash_{\mathbf{S}} \beta \text { and } \beta \vdash_{\mathbf{S}} \varphi \text {, then } \alpha \vdash_{\mathbf{S}} \varphi
$$

is a particular case of the property of cut with $\Sigma=\{\beta\}$ and $\Gamma=\{\alpha\}$.

${ }^{9}$ Compactness Theorem. If $\Sigma$ is a set of formulas of a first order language with equality such that every finite subset of $\Sigma$ is satisfiable, then $\Sigma$ is also satisfiable. Cf. Malitz 1979, pp. 162-165 and Amor 1999, pp. 28-36.

${ }^{10}$ That is, if $\exists x \varphi(x) \in \Omega$ then there is a constant $c$ (the existential witness), such that $\varphi(c) \in \Omega$. 
By the simple fact that by definition the derivations are finite, it follows that the used hypotheses in a derivation constitute a finite set, although there may be an infinity of unused hypotheses. From here one gets the so-called finiteness lemma for the derivation, that is fulfilled in any axiomatic system $\mathbf{S}$.

c) Finiteness Lemma. If $\mathbf{S}$ is an axiomatic system and $\Sigma \cup\{\varphi\}$ is a set of formulas, then: $\Sigma \vdash_{\mathrm{s}} \varphi$ if and only if there is a set $\Gamma \subseteq \Sigma, \Gamma$ finite, such that $\Gamma \vdash_{\mathrm{s}} \varphi$.

Some of these syntactic properties will be used from now on, appealing to any of them only as "syntactic properties".

Another fundamental logical notion of syntactic type is the notion of consistency of sets of formulas. A set of formulas $\Sigma$ is consistent with respect to an axiomatic system $\mathbf{S}$ (or $\mathbf{s}-$ consistent) if it is not derived from $\Sigma$ in the system $\mathbf{S}$, a formula and its negation; that is, it is not possible to derive a contradiction. Note that this concept is not absolute in general, but relative to the axiomatic system, which I deal with. ${ }^{11} \mathrm{Nev}$ ertheless, it is interesting to note that this is not clarified in most classical texts. ${ }^{12}$ For this reason, in general it should be spoken about s-consistency and not about consistency, unless the relativity has been clarified and it is obvious to what system I refer to.

To finish this section I will give two properties that refer to axiomatic systems. These properties are not fulfilled in all systems. Actually, the fact that a system satisfies them means that it has fundamental properties for its adequation with logical consequence.

The metalogical relation between the logical notions of derivability of a formula $\beta$ from a formula $\alpha$ in an axiomatic system

${ }^{11}$ If $\Sigma$ is a set of formulas to which $\alpha$ and $\neg \alpha$ both pertain, then $\Sigma$ is inconsistent in an absolute way, because in that case within any system a contradiction is derived. But it is possible for some set of formulas, to be consistent with respect to an axiomatic system and inconsistent with respect to another one. In these cases, consistency of sets of formulas is relative to the system Amor 2001, pp. 66-67.

${ }^{12}$ Cf. Mendelson 1997, p. 72, Enderton 1972, pp. 112 and 128, Manzano 1989, p. 115. 
$\mathbf{S}$ (that is, $\alpha \vdash_{\mathbf{S}} \beta$ ), and that of proving without hypotheses the formula $(\alpha \rightarrow \beta)$ in $\mathbf{S}$ (that is, $\vdash_{\mathbf{S}}(\alpha \rightarrow \beta)$ ), turns out to be very important in classical logic. For that reason, it will be analyzed with some detail and then I will give some results that involve it.

First I generalize this relation considering moreover any set $\Sigma$ of possible extra hypotheses; that is, I analyze the relation between $\Sigma, \alpha \vdash_{\mathbf{S}} \beta$ and $\Sigma \vdash_{\mathbf{S}}(\alpha \rightarrow \beta)$.

It is easy to observe that the metalogical implication:

$$
\text { If } \Sigma \vdash_{\mathbf{S}}(\alpha \rightarrow \beta) \text {, then } \Sigma, \alpha \vdash_{\mathbf{S}} \beta
$$

is equivalent to the fact that the system has the rule of Modus Ponens (MP), as an original rule of the system $\mathbf{S}$ or as a derived rule of inference of the system. ${ }^{13}$ These ideas will be precise with the following definition and the following lemma.

Definition. Let $\mathbf{S}$ be an axiomatic system. I say that $\mathbf{S}$ satisfies Modus Ponens (MP) if and only if for all $\Gamma, \alpha$ and $\beta$, the following is satisfied:

$$
\Gamma, \alpha,(\alpha \rightarrow \beta) \vdash_{\mathbf{s}} \beta \text {. }
$$

Lemma. Let $\mathbf{S}$ be an axiomatic system. Then: $\mathbf{S}$ satisfies MP if and only if for any set of formulas $\Sigma$ and any formulas $\alpha, \beta$ : if $\Sigma \vDash_{\mathbf{S}}(a \rightarrow \beta)$ then $\Sigma, \alpha \vDash_{\mathbf{S}} \beta$.

Proof. Let $\mathbf{S}$ be an axiomatic system.

$\Rightarrow)$ Suppose that $\mathbf{S}$ satisfies MP. Let $\Sigma, \alpha, \beta$ be any formulas.

(1) $\Gamma, \alpha,(\alpha \rightarrow \beta) \vdash_{\mathbf{S}} \beta \quad$ supposition: for all $\Gamma, \alpha, \beta$

(2) $\Sigma \vdash_{\mathbf{S}}(\alpha \rightarrow \beta) \quad$ supposition

(3) $\Sigma, \alpha \vdash_{\mathbf{S}}(\alpha \rightarrow \beta) \quad$ syntactic property: monotony

(4) $\Sigma, \alpha,(\alpha \rightarrow \beta) \vdash_{\mathbf{S}} \beta \quad$ from (1), with $\Gamma=\Sigma$

(5) $\Sigma, \alpha \vdash_{\mathbf{S}} \beta \quad$ from (3) and (4) by cut

So, from (2)-(5), I have that: $\Sigma \vdash_{\mathbf{S}}(\alpha \rightarrow \beta) \Rightarrow \Sigma, \alpha \vdash_{\mathbf{S}} \beta$.

${ }^{13}$ If a rule of inference $\mathrm{R}$ has the form: $\alpha_{1}, \ldots, \alpha_{n} / \beta$; that is, to obtain $\beta$ from $\alpha_{1}, \ldots, \alpha_{n}$, then $\mathrm{R}$ is a derived rule in the system $\mathbf{S}$ if and only if $\mathrm{R}$ is not an original rule of the set RI of inference rules of $\mathbf{S}$ and $\alpha_{1}, \ldots, \alpha_{n} \vdash_{\mathbf{S}} \beta$. 

$\beta$.

$\Leftarrow)$ Suppose now: $\Sigma \vdash_{\mathbf{S}}(\alpha \rightarrow \beta) \Rightarrow \Sigma, \alpha \vdash_{\mathbf{S}} \beta$, for all $\Sigma, \alpha$,

Let $\Gamma, \alpha, \beta$, be any formulas.

(1) $\Sigma \vdash_{\mathbf{S}}(\alpha \rightarrow \beta) \Rightarrow \Sigma, \alpha \vdash_{\mathbf{S}} \beta \quad$ supposition: for all

$\Sigma, \alpha, \beta$

(2) $\Gamma, \alpha,(\alpha \rightarrow \beta) \vdash_{\mathbf{S}}(\alpha \rightarrow \beta) \quad$ reflexivity and

(3) $\Gamma, \alpha,(\alpha \rightarrow \beta), \alpha \vdash_{\mathbf{s}} \beta \quad$ (2) and (1) with $\Sigma=$

$\Gamma \cup\{\alpha,(\alpha \rightarrow \beta)\}$

(4) $\Gamma, \alpha,(\alpha \rightarrow \beta) \vdash_{\mathbf{S}} \beta \quad$ eliminating the repeated $\alpha$

So, by (4), S satisfies MP.

The inverse metalogical implication, known as the Deduction Metatheorem, is a very special property of an axiomatic system $\mathbf{S}$. This is because moreover and independently of giving a mechanism for the simplification of the proofs of formulas having form of implications, ${ }^{14}$ it has a great theoretical importance because it is related to the extended correctness property of the system, as I will see in the following section on the metalogical results.

Observe that the Deduction Metatheorem states that a conditional is derivable from a set of formulas, if its consequent is derivable from the set of formulas augmented with the antecedent. More precisely:

Deduction Metatheorem (DMT) for S. For any set of formulas $\Sigma$ and any formulas $\alpha$ and $\beta$, it is fulfilled that:

$$
\text { if } \Sigma, \alpha \vdash_{\mathbf{S}} \beta \text {, then } \Sigma \vdash_{\mathbf{S}}(\alpha \rightarrow \beta) \text {. }
$$

\section{Metalogical Results}

To begin this section I make precise what I will understand by the property of extended correctness and completeness in first order logic with equality.

\footnotetext{
${ }^{14}$ Because instead of having to derive $(\alpha \rightarrow \beta)$, I can additionally suppose $\alpha$ as an extra hypothesis and then derive $\beta$.
} 
An axiomatic system $\mathbf{S}$ satisfies the extended correctness property if any formula obtained with a derivation in $\mathbf{S}$ from $\Sigma$, is a logical consequence of $\Sigma$. Conversely, a system $\mathbf{S}$ satisfies the extended completeness property if all the logical consequences of $\Sigma$ can be obtained with the process of derivation from $\Sigma$ in $\mathbf{S}$. In both concepts, the restricted version refers to the particular case in which there are no formulas in $\Sigma$; that is, $\Sigma$ is empty or there are no hypotheses.

The extended correctness and completeness theorem asserts the existence of an axiomatic system that satisfies both the extended correctness property and the extended completeness property.

With my present characterization of logical consequence of section 2, there are deductive systems $\mathbf{S}$ which satisfy both extended correctness and extended completeness: $\Gamma \vDash \varphi$ if and only if $\Gamma \vdash_{\mathbf{s}} \varphi$. An example is the system of Enderton. ${ }^{15}$ These systems, according to clause c) of section 2 , must satisfy that:

$$
\text { if } \Gamma, \alpha \vdash_{\mathbf{S}} \beta \text { then } \Gamma \vdash_{\mathbf{s}}(\alpha \rightarrow \beta) \text {. }
$$

The standard inference rule of Universal Generalization $(\forall x \varphi$ follows from $\varphi)^{16}$ is not a counterexample to the existence of deductive systems which satisfy extended correctness, but is an example of a not very good rule because it generates derivations that are not logical consequences, for example $P(x) \vdash \forall x P(x)$, but $P(x) \not \forall \forall x P(x)$.

In this section I will show two logical relations between logical properties of the system. The first one is a relation between the property of satisfying Modus Ponens and the property of extended completeness; the second one is a relation between the DMT and the property of extended correctness; both for systems that satisfy the restricted correctness and completeness properties. The discovery of these general relations helped us to find that a direct way of proving the extended correctness and the extended completeness of a particular system that satisfies restricted correctness and completeness, had to be by proving

${ }^{15}$ Cf. Enderton 2001, pp. 131-141.

${ }^{16}$ Cf. Mendelson 1987, p. 56. 
that the system satisfies both Modus Ponens and the Deduction Metatheorem. I will state next the first result, on the logical relation between two logical properties of axiomatic systems: that of satisfying MP and that of satisfying extended completeness. This metalogical relation is expressed with the following proposition.

Proposition 1. If $\mathbf{S}$ is an axiomatic system that satisfies restricted completeness, then: $\mathbf{S}$ satisfies extended completeness if and only if $\mathbf{S}$ satisfies MP.

Proof. Let $\mathbf{S}$ be a formal system that satisfies restricted completeness (in other words, $\vDash \alpha \Rightarrow \vdash_{\mathbf{S}} \alpha$ ).

$\Rightarrow)$ Suppose that $\mathbf{S}$ satisfies extended completeness $(\Sigma \vDash \alpha \Rightarrow$ $\Sigma \vdash_{\mathrm{S}} \alpha$ ). Let $\Gamma$ be a set of formulas and $\alpha, \beta$ formulas. It is immediate that:

(1) $\Gamma, \alpha,(\alpha \rightarrow \beta) \vDash \beta \quad$ semantic property e)

(2) $\Gamma, \alpha,(\alpha \rightarrow \beta) \vdash_{\mathbf{S}} \beta \quad$ extended completeness of $\mathbf{S}$

So, by (2), $\mathbf{S}$ satisfies MP.

$\Leftarrow)$ Suppose now that $\mathbf{S}$ satisfies $\mathrm{MP}\left(\Gamma, \alpha,(\alpha \rightarrow \beta) \vdash_{\mathbf{S}} \beta\right)$. Let $\Sigma$ be a set of formulas and $\alpha$ a formula, such that:

(1) $\Sigma \vDash \alpha$

(2) There is a finite list

supposition

$\gamma_{1}, \ldots, \gamma_{n}$ of formulas

of $\Sigma$, such that $\gamma_{1}, \ldots, \gamma_{n} \vDash \alpha \quad$ Compactness Theorem

(3) $\vDash\left(\gamma_{1} \rightarrow\left(\gamma_{2} \rightarrow \ldots \rightarrow\right.\right.$

$\left.\left.\left(\gamma_{n} \rightarrow \alpha\right)\right) \ldots\right)$

(4) $\vdash_{\mathbf{S}}\left(\gamma_{1} \rightarrow\left(\gamma_{2} \rightarrow \ldots \rightarrow\right.\right.$

$\left.\left.\left(\gamma_{n} \rightarrow \alpha\right)\right) \ldots\right)$

semantic property ${ }^{17}$

restricted completeness

of $\mathbf{S}$

(5) $\gamma_{1}, \ldots, \gamma_{n} \vdash_{\mathbf{s}} \alpha$

$\mathbf{S}$ satisfy MP and

Lemma $n$ times

(6) $\Sigma \vdash_{\mathbf{s}} \alpha$ syntactic property a) and $\left\{\gamma_{1}, \ldots, \gamma_{n}\right\} \subseteq \Sigma$

So, by (1)-(6), S satisfies extended completeness.

${ }^{17}$ Applying the semantic property c), $n$ times. 
Then, given an axiomatic system that satisfies restricted completeness, a necessary and sufficient condition to satisfy extended completeness is to satisfy MP.

It follows from Proposition 1 that for an axiomatic system, to satisfy extended completeness and to satisfy both restricted completeness and Modus Ponens are equivalent properties.

I observe then, that to satisfy MP is so important that if a system with restricted completeness satisfies it, that is enough for satisfying extended completeness. But if a system doesn't satisfy it, then although it satisfies restricted correctness and completeness, it will never satisfy extended completeness. An example of this is Malitz's system. ${ }^{18}$ This is a system that satisfies restricted completeness and correctness and nevertheless it doesn't satisfy extended completeness and the reason of that is that it doesn't satisfy MP.

The second result is on a metalogical relation between the DMT and the property of extended correctness and it is stated with the following proposition.

Proposition 2. If $\mathbf{S}$ is an axiomatic system that satisfies restricted correctness and extended completeness, then: $\mathbf{S}$ satisfies extended correctness if and only if $\mathbf{S}$ satisfies the DMT.

Proof. Let $\mathbf{S}$ be an axiomatic system that satisfies restricted correctness and extended completeness.

$\Rightarrow)$ Suppose that $\mathbf{S}$ satisfies extended correctness $\left(\Sigma \vdash_{\mathbf{S}} \alpha \Rightarrow\right.$ $\Sigma \vDash \alpha$ ). Let $\Gamma$ be a set of formulas, $\alpha$ and $\beta$ formulas such that:

(1) $\Gamma, \alpha \vdash_{\mathbf{S}} \beta$

(2) $\Gamma, \alpha \vDash \beta$

(3) $\Gamma \vDash(\alpha \rightarrow \beta)$

(4) There is a finite list $\gamma_{1}, \ldots, \gamma_{n}$ of formulas of $\Gamma$ such that

$\gamma_{1}, \ldots, \gamma_{n} \vDash(\alpha \rightarrow \beta)$

(5) $\gamma_{1}, \ldots, \gamma_{n} \vdash_{\mathbf{s}}(\alpha \rightarrow \beta)$

(6) $\Gamma \vdash_{\mathbf{s}}(\alpha \rightarrow \beta)$ supposition

extended correctness

semantic property c)

Compactness Theorem extended completeness in $\mathbf{S}$ syntactic property a) and $\left\{\gamma_{1}, \ldots, \gamma_{n}\right\} \subseteq \Gamma$

${ }^{18}$ Cf. Malitz 1979, p. 188 and Amor 1999, p. 110. 
So, by (1)-(6), S fulfills the DMT.

$\Leftarrow$ ) Suppose now that $\mathbf{S}$ satisfies the DMT (if $\Gamma, \alpha \vdash_{\mathbf{S}} \beta$ then $\left.\Gamma \vdash_{\mathbf{S}}(\alpha \rightarrow \beta)\right)$. Let $\Sigma$ be a set of formulas and $\alpha$, a formula such that:

(1) $\Sigma \vdash_{\mathbf{S}} \alpha$

(2) There is a finite list $\gamma_{1}, \ldots, \gamma_{n}$ of formulas of $\Sigma$, such that

$\gamma_{1}, \ldots, \gamma_{n} \vdash_{\mathbf{s}} \alpha$

(3) $\vdash_{\mathbf{s}}\left(\gamma_{1} \rightarrow\right.$

$\left.\left(\gamma_{2} \rightarrow \ldots \rightarrow\left(\gamma_{n} \rightarrow \alpha\right)\right) \ldots\right)$

(4) $\vDash\left(\gamma_{1} \rightarrow\right.$

$\left.\left(\gamma_{2} \rightarrow \ldots \rightarrow\left(\gamma_{n} \rightarrow \alpha\right)\right) \ldots\right)$

(5) $\gamma_{1}, \ldots, \gamma_{n} \vDash \alpha$

(6) $\Sigma \vDash \alpha$ supposition

Finiteness Lemma ${ }^{19}$

DMT $n$ times

restricted correctness of $\mathbf{S}$

semantic property ${ }^{20}$

semantic property a) and $\left\{\gamma_{1}, \ldots, \gamma_{n}\right\} \subseteq \Sigma$

So, by (1)-(6), S fulfills extended correctness.

Of course, Proposition 2 could be proved by assuming restricted completeness plus MP instead of extended completeness, because these two properties are equivalent as we saw after Proposition 1.

We can observe then that the DMT is so important that if a system with restricted correctness satisfies it, that is enough for satisfying extended correctness, but if a system doesn't satisfy it, although it fulfills restricted correctness-completeness and Modus Ponens, it will never satisfy extended correctness. An example of this is the system that is obtained from that of Malitz ${ }^{21}$ by adding to it MP as a new rule of inference. This is a system that satisfies extended completeness and restricted correctness and nevertheless it does not satisfy extended correctness and the reason is that it does not satisfy the DMT.

I present finally as a corollary of the two main propositions of this section, a structural characterization of extended

\footnotetext{
${ }^{19}$ Syntactic property c).

${ }^{20}$ Applying the semantic property c), $n$ times.

${ }^{21}$ Cf. Malitz 1979, p. 188 and Amor 1999, p. 110.
} 
correctness-completeness for systems that satisfy the corresponding restricted properties.

Corollary. If $\mathbf{S}$ is an axiomatic system that satisfies restricted correctness-completeness, then: $\mathbf{S}$ satisfies extended correctnesscompleteness if and only if $\mathbf{S}$ satisfies MP and DMT.

\section{Conclusions}

In this paper I have given some elementary semantic properties that are used in various results. I have also given a definition of axiomatic system and some basic syntactic properties that are used too in various results. Then I presented the notion of consistency of a set of formulas, relative to an axiomatic system and finished explaining the meaning of an axiomatic system to satisfy Modus Ponens and that of a system to satisfy the Deduction Metatheorem. I presented and proved, by using the compactness theorem, the metalogical results that show the property of satisfying Modus Ponens as a necessary and sufficient condition for the extended completeness of the system, and to the Deduction Metatheorem as a necessary and sufficient condition for the extended correctness of the system. Both supposing that the system satisfies the corresponding restricted properties. These results show how to find a way of proving the extended correctness and the extended completeness of a particular axiomatic system, if it satisfies the restricted correctness and completeness. That is, I showed that the system had necessarily to satisfy Modus Ponens and the Deduction Metatheorem. ${ }^{22}$ Finally these metalogical results show that the choice of this rule of inference (MP) and of this Metatheorem (DMT) for a particular axiomatic system are not a matter of personal liking or of convenience. As a matter of fact they can be considered as intrinsic structural properties that are essential in classical logic, and that play a fundamental role in the fulfilling of the Extended Correctness and Completeness theorem of an axiomatic system.

${ }^{22}$ Cf. Amor 2001. 


\section{REFERENCES}

Aliseda, A., 1999, Reseña de Compacidad en la lógica de primer orden y su relación con el teorema de completud, de J.A. Amor, Crítica, Revista Hispanoamericana de Filosofía, vol. XXXI, no. 93, pp. 117-124.

Amor, J.A., 2001, Relaciones metalógicas entre compacidad y completud: una prueba semántica de completud en lógica clásica (tesis de doctorado), unAm, México.

__ 1999, Compacidad en la lógica de primer orden y su relación con el teorema de completud, Coordinación de Servicios Editoriales, Facultad de Ciencias/UnAM, México.

Enderton, H.B., 2001, A Mathematical Introduction to Logic, 2nd ed., Academic Press, New York.

Gödel, K., 1986, Collected Works, in Solomon Feferman (ed.), vol. I, Oxford University Press, Oxford.

—_, 1981, Obras completas, in Jesús Mosterín (ed.), Alianza Universidad, Madrid.

- 1930a, The Completeness of the Axioms of the Functional Calculus of Logic, in Gödel 1986, pp. 103-123.

——, 1930b, La suficiencia de los axiomas del cálculo lógico funcional, in Gödel 1981, pp. 20-34.

- 1930c, The Completeness of the Axioms of the Functional Calculus of Logic, in van Heijenoort 1967, pp. 582-591.

- 1929, On the Completeness of the Calculus of Logic, in Gödel 1986, pp. 61-101.

Malitz, J., 1979, Introduction to Mathematical Logic, Model Theory (Part III), Springer-Verlag, New York.

Manzano, M., 1989, Teoría de modelos, Alianza Editorial, Madrid.

Mendelson, E., 1987, Introduction to Mathematical Logic, 3rd ed., Wadsworth and Brooks, California.

Tarski, A., 1935, Der Wahrheitsbegriff in den formaliiferten Sprachen, Studia Philos., Warsaw, 1, pp. 261-405. [English version: Logic, Semantic and Metamathematics, Oxford, 1956, pp. 152-278.]

Van Heijenoort, J., 1967, From Frege to Gödel, A Source Book in Mathematical Logic, 1879-1931, Harvard University Press, Cambridge.

Received December 4, 2002; revised July 17, 2003; accepted August 13, 2003. 